\title{
Shot reproducibility of the self-magnetic-pinch diode at 4.5 MV
}

\author{
Nichelle Bennett, M. Dale Crain, Darryl W. Droemer, Raymond E. Gignac, Greg Lare, \\ Isidro Molina, Robert Obregon, Chase C. Smith, and Frank L. Wilkins \\ National Security Technologies, LLC, Las Vegas, Nevada 89193, USA \\ Dale R. Welch \\ Voss Scientific, LLC, Albuquerque, New Mexico 87108, USA
}

\begin{abstract}
Steve Cordova, Manuel L. Gallegos, Mark D. Johnston, Mark L. Kiefer, Joshua J. Leckbee, Michael G. Mazarakis, Dan Nielsen, Timothy J. Renk, Tobias Romero,

Timothy J. Webb, and Derek Ziska

Sandia National Laboratories, Albuquerque, New Mexico 87185, USA
\end{abstract}

(Received 15 November 2013; published 27 May 2014)

\begin{abstract}
In experiments conducted at Sandia National Laboratories' RITS-6 accelerator, the self-magnetic-pinch diode exhibits significant shot-to-shot variability. Specifically, for identical hardware operated at the same voltage, some shots exhibit a catastrophic drop in diode impedance. A study is underway to identify sources of shot-to-shot variations which correlate with diode impedance collapse. The scope of this report is limited to data collected at 4.5-MV peak voltage and sources of variability which occur away from the diode, such as sheath electron emission and trajectories, variations in pulsed power, load and transmission line alignment, and different field shapers. We find no changes in the transmission line hardware, alignment, or hardware preparation methods which correlate with impedance collapse. However, in classifying good versus poor shots, we find that there is not a continuous spectrum of diode impedance behavior but that the good and poor shots can be grouped into two distinct impedance profiles. In poor shots, the sheath current in the load region falls from $16 \%-30 \%$ of the total current to less than $10 \%$. This result will form the basis of a follow-up study focusing on the variability resulting from diode physics.
\end{abstract}

DOI: 10.1103/PhysRevSTAB.17.050401

PACS numbers: 41.85.Ja, 52.25.Fi, 52.65.Rr, 52.65.Ww

\section{INTRODUCTION}

Radiation pulse reproducibility is critically important for the radiographic sources under development at Sandia National Laboratories. To determine the suitability of the self-magnetic-pinch (SMP) diode [1-6] as a radiographic source, an experimental campaign was conducted on Sandia's RITS-6 accelerator [7-9] to quantify its shot-toshot variation. The accelerator operated at $4.5 \mathrm{MV}$ and the SMP diode was fielded with changes only to the anodecathode (AK) gap and cathode needle radius $\left(r_{c}\right)$. In this report, a diode geometry is labeled by the needle diameter and AK gap width, such that a 7-mm-diameter cathode fielded with a 7-mm AK gap width is a 7-7 shot.

Variations in SMP diode performance occur in spot size, radiation pulse width, and impedance lifetime. In normal operation, this diode has a decreasing impedance related to anode plasma expansion [10]. Occasionally this diode experiences a more rapid impedance drop $(>0.5 \Omega / \mathrm{ns})$

Published by the American Physical Society under the terms of the Creative Commons Attribution 3.0 License. Further distribution of this work must maintain attribution to the author(s) and the published article's title, journal citation, and DOI. resulting in a radiographically poor shot. We wish to identify sources of shot variability which correlate with diode impedance collapse. To eliminate unintended spacecharged-limited electron emission as a source, only data from a shot series conducted with a 4.5-MV peak voltage are considered.

A schematic of the downstream end of the accelerator, including the diode, is shown in Fig. 1. Possible sources of variation between shots with identical hardware and geometry include (i) sheath electron emission and trajectories, (ii) variations in pulsed power, (iii) dustbin and transmission line misalignment, (iv) different field shapers, (v) diode misalignment, (vi) nonuniform electron emission from the cathode needle, (vii) nonuniform emission from the faceplate, and (viii) an unidentified plasma-induced impedance collapse mechanisms. In this paper, we examine only the sources which occur away from the diode (the first four).

To address the first two items above, we investigate the possibility of shot-to-shot variations in the electron emission along the magnetically insulated transmission line (MITL). We simultaneously look at the dynamics of sheath electrons in the dustbin, which are related to variations in emission and to the load impedance. Since changes in hardware preparation methods could alter the cathode 


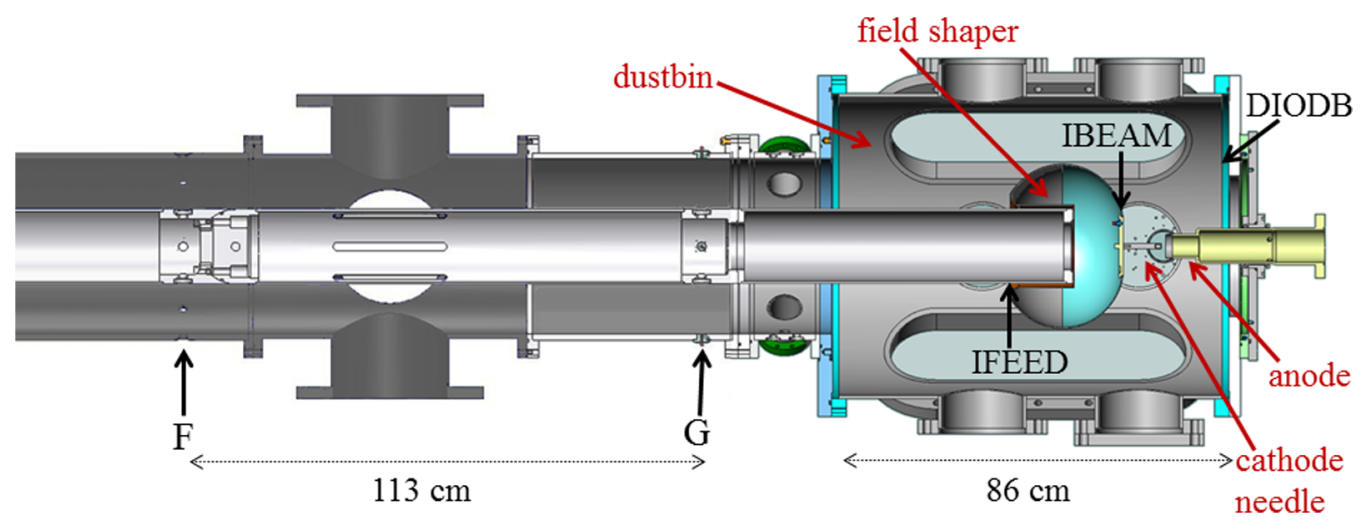

FIG. 1. Diagram of the downstream portion of the RITS-6 accelerator. The B-dot current monitors at positions F, IFEED, IBEAM, and DIODB are labeled.

surface and its electric field stress thresholds for electron emission, simulations are used to determine the thresholds for a sample of shots. The measured forward-going voltage pulses are used as input to the simulations to enable direct comparisons to shot data. Since the simulation of each shot takes four days on 20 processors, the statistics of this analysis are limited. The shots are sampled from March through August of 2013. The results, presented in Sec. III, show consistent emission thresholds and a consistent pattern of electron flow in the dustbin region from shot to shot.
Shot-to-shot variations in pulsed power are examined in Sec. IV. The anode currents in the MITL are compared for 99 shots to determine if poor diode performance is correlated with peak injected power or pulse rise. Poor diode performance is indicated by an above-average reduction in diode impedance, and subsequent rise in the cathode current, as discussed in Sec. II. The peak anode currents and pulse rises for good shots are fit to Gaussian distributions. The goodness of fit of the poor shots to these distributions indicates that they are not systematically related to either of these pulsed-power parameters.
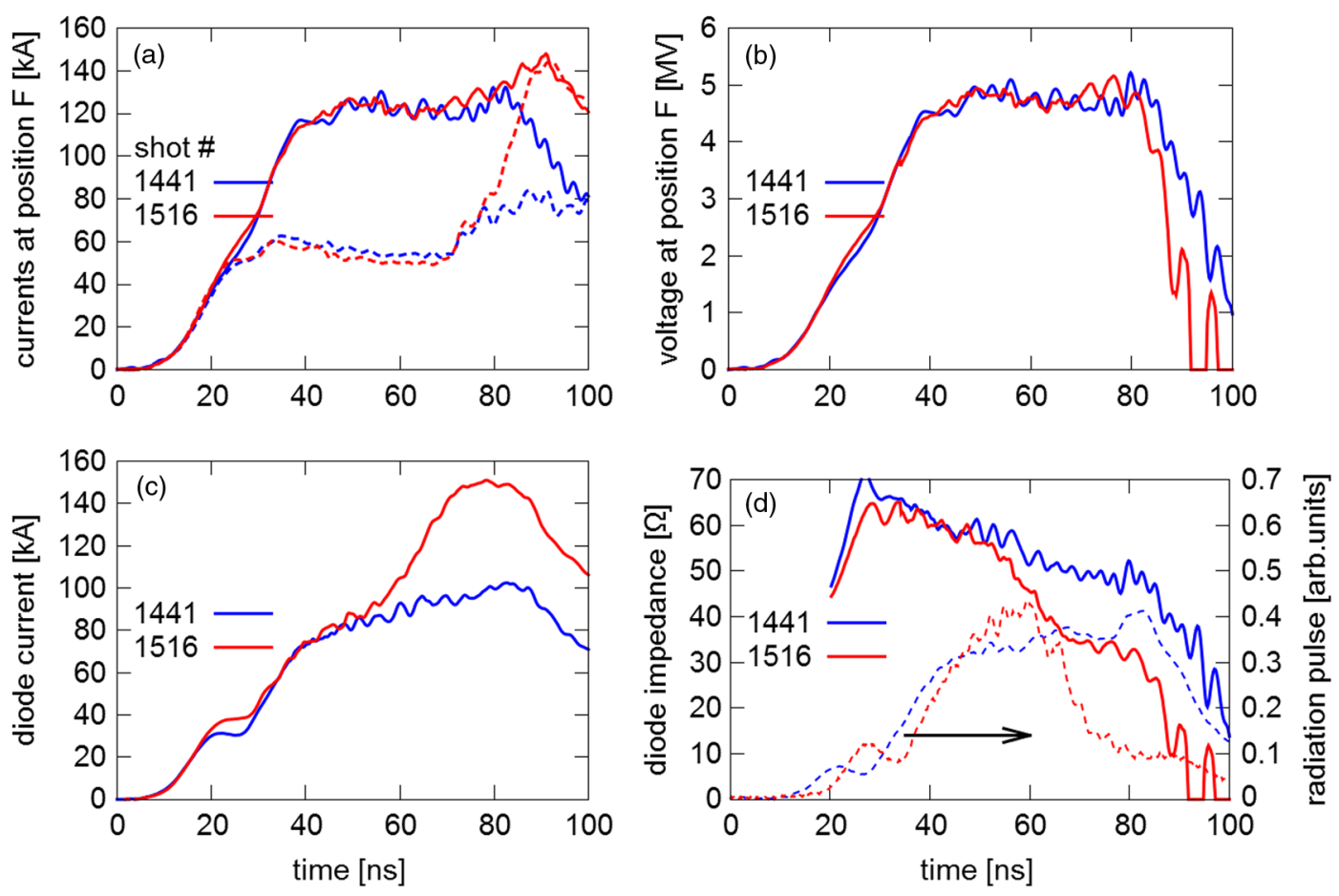

FIG. 2. Traces of the currents, voltage, diode impedance, and radiation pulse for two SMP diode shots with a 7-mm diameter cathode needle and a 7-mm AK gap. Shot 1441 (blue) demonstrates the desired diode behavior while Shot 1516 (red) has a premature radiation pulse termination due to a rapid drop in diode impedance. The IF (dashed) and OF (solid) currents are show in (a). The voltage at position $\mathrm{F}$ is shown in (b). The diode current is shown in (c). The diode impedance (solid) and radiation pulse (dashed) are shown in (d). 

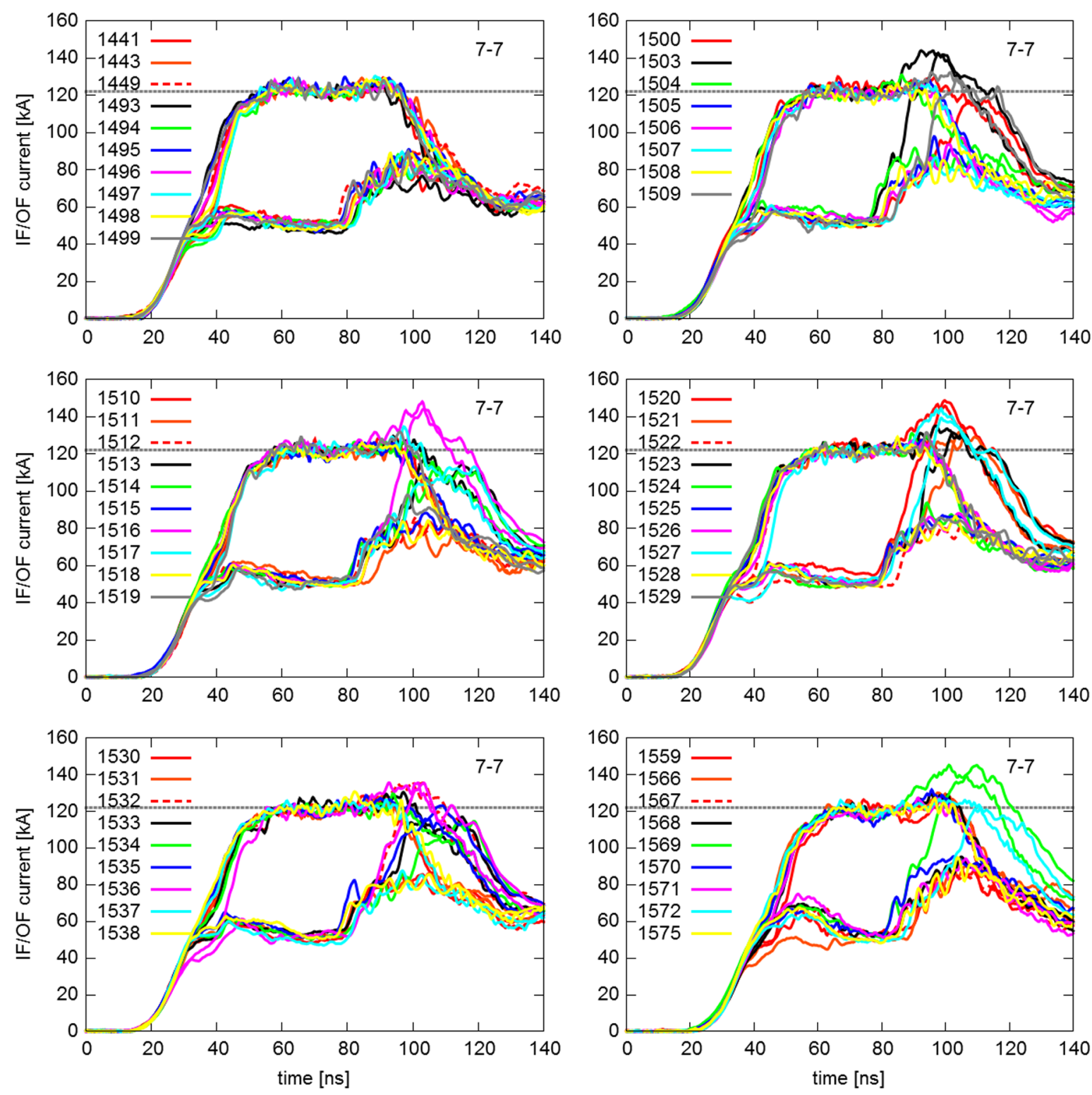

FIG. 3. The IF and OF currents for SMP diode shots with a 7-mm diameter cathode needle and a 7-mm AK gap. The gray dotted lines are the average $\mathrm{OF}$ in the first $10 \mathrm{~ns}$ at peak power.

The effects of MITL and dustbin alignment are treated similarly. Misalignment could cause azimuthally asymmetric currents, which might affect the diode. Asymmetries for the MITL and dustbin B-dot probes are calculated and sorted by diode performance in Sec. V. No correlation is found between the level of azimuthal asymmetry and poor shots.

Of hardware correlations, we found that one of the six field shapers used is more highly correlated with poor shots. However, as shown in Sec. V, poor shots are recorded for each, so field shaper type is unlikely to be the primary cause of impedance collapse.

Poor shots are identified in Sec. II by a larger-thanaverage rise in their cathode currents in the MITL which is a result of lower load impedance. The motivation that this is due to diode impedance collapse is provided in Sec. VI. The diode impedance profiles form a bimodal distribution for good and poor shots.

The diode currents are compared to the theoretical critical current in Sec. VII. We modify this theory to account for a time-dependent AK gap width and calculate a gap closure velocity of approximately $v_{p}=1 \mathrm{~cm} / \mu \mathrm{s}$. The theory is well-matched to data, which exhibit the expected scaling with cathode and AK gap size.

\section{IDENTIFYING POOR RADIOGRAPHIC SHOTS}

Poor radiographic performance of a diode is the result of the electron beam having a large or wandering focus at the 
converter, a low diode voltage, or a low usable beam current. When the SMP diode fails, it experiences an increase in local current, either due to a rapid drop in impedance or because it no longer obeys the standard critical-current equation (see for example Ref. [3]). The drop in the impedance presumably disrupts the beam focus because the radiation output is truncated.

The diode failure is observed upstream as a decrease in load impedance below what is expected for the dustbin/ field shaper and diode geometries. The SMP diode as fielded on RITS-6 is undermatched to the MITL, so some sheath current is retrapped in the cathode for emission in the diode. The amount of retrapping is much larger when the diode fails. Figure 2 illustrates this with a comparison of data from two SMP-diode shots, one with the desired diode impedance lifetime (Shot 1441) and one with a poor impedance lifetime (Shot 1516). The transmission line currents plotted in Fig. 2(a) are measured at position F, which is defined in Sec. III. The anode and cathode currents at F, labeled OF and IF, respectively, are plotted for both shots. The voltage calculated from OF and IF $[11,12]$ is shown in Fig. 2(b). The rise in the cathode current in the second half of the pulse marks the arrival of the retrapping wave. The diode current for Shot 1441, shown in Fig. 2(c), represents the normal impedance decrease as shown in Fig. 2(d). The diode impedance is estimated using the diode current and the voltage from F. The diode current for Shot 1516 increases above the normal range by approximately midway through the pulse. This is shown as the more rapid decrease in impedance in Fig. 2(d) and the very high retrapped cathode current in Fig. 2(a). The effect of the impedance drop on the radiation output is also shown in Fig. 2(d). The forward-going x-rays are recorded using a PIN diode. The radiation pulse from Shot 1516 is shorter and correlated with a drop in the diode impedance.

With this demonstrated relationship between the transmission line currents and the diode impedance, examining a large number of shots shows that normal impedance behavior is relatively uniform while failures have varying rates of impedance fall. This is seen in the $\mathrm{OF}$ and $\mathrm{IF}$ currents plotted in Fig. 3 for 56 of the shots analyzed. The diode geometries are labeled in each figure. The cathode current after retrapping differs by geometry, or baseline diode impedance, but is still noticeably larger in poor shots.

\section{MITL ELECTRON EMISSION THRESHOLDS}

Changes in electron emission and trajectories in the transmission line may impact diode performance, particularly if sheath electrons are impacting the anode near the diode. To determine the consistency of the hardware preparations and the operating conditions of the MITL, dustbin, and field shaper, emission thresholds are determined in simulations of specific shots. For each shot considered, the forward-going voltage calculated at position $\mathrm{F}$ is used as the injected voltage wave in its corresponding simulation. The simulation parameters, such as electron emission thresholds, are adjusted to achieve the best match to the measured B-dot currents at five locations. Variations in these model parameters from simulation to simulation would indicate shot-to-shot variations in the transmission line and dustbin.

The B-dot probe positions analyzed are IF and $O F$ The inner and outer currents at position $\mathrm{F}$, located $1.16 \mathrm{~m}$ upstream from the dustbin entrance. IFEED The cathode current immediately upstream from the field shaper. IBEAM The cathode current inside a $6.35-\mathrm{cm}$ radius on the field-shaper face plate. This includes the beam current and a limited region of possible face-plate emission. $D I O D B$ The anode current inside an $11-\mathrm{cm}$ radius on the end plate.

The measured boundary current profile at position $\mathrm{F}$ indicates that the cathode emits earlier downstream than upstream. This is consistent with the location of pump-out ports in the center conductor. We approximate this effect of higher local field stress by reducing the electron emission threshold in the region of the pump-out ports (see Sec. III A). Using the same set of emission thresholds for all simulations, the currents are all in reasonable agreement with their respective shot data, shown in detail in Secs. III B and III C. The onset of emission at position F, the sheath electron trajectories in the dustbin, and the retrapping rate on the field shaper are well modeled. This model uniformity is significant because even a $5 \%$ change in the emission threshold in the MITL caused a noticeable change in cathode current. Therefore, the MITL conditions (cleaning process, application of Aerodag, alignment) appears consistent from shot to shot.

\section{A. Simulation details}

All simulations are performed in 2D cylindrical coordinates using the fully-relativistic electromagnetic particlein-cell code LSP [13]. All simulations have the same geometry, covering the dustbin region and $1.7 \mathrm{~m}$ of the MITL upstream of the dustbin. The common simulation geometry is illustrated in Fig. 4. The accelerator cavities are omitted so that measured forward-going voltage pulses may be used to drive the simulations. This enables direct

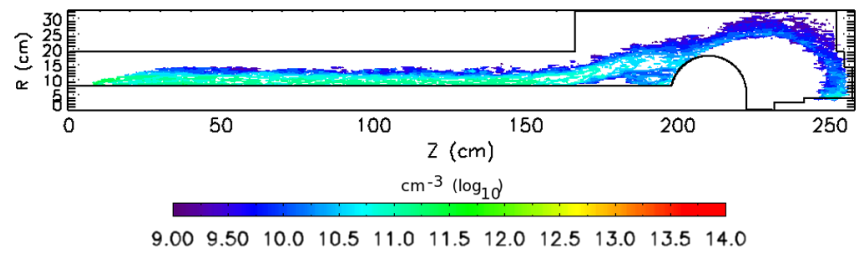

FIG. 4. Simulation geometry of the downstream section of the RITS-6 accelerator, including the dustbin and diode. Contours of the sheath electron density at peak power are also shown on a logarithmic scale. 


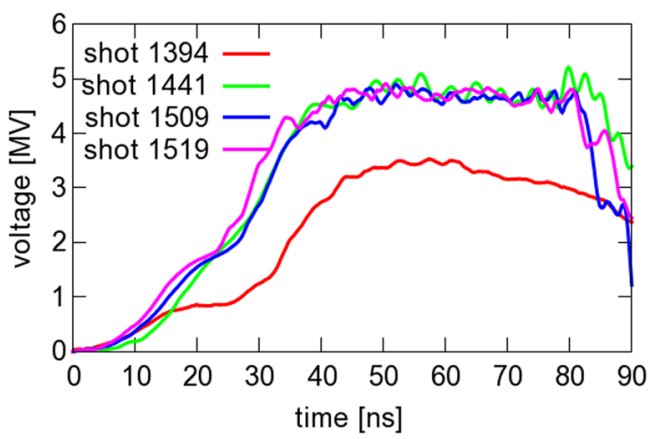

FIG. 5. Measured voltages used to drive four simulations of the SMP diode.

comparisons between currents generated in simulations and data.

In Fig. 4, the dustbin covers $166<z<257 \mathrm{~cm}$ and the field shaper is located from $z=197.5 \mathrm{~cm}$ to $z=222.5 \mathrm{~cm}$. The subcell model is used for the field shaper to reduce the effect of field enhancement due to the stair-stepped representation of the curved surface. Probe position $\mathrm{F}$ is located at $z=50.0 \mathrm{~cm}$, IFEED is at $z=197.5 \mathrm{~cm}$, IBEAM is at $(6.35,222.65)$, and DIODB is at $(11.0,257.0)$.

The diode is modeled with the actual SMP diode dimensions fielded, but with the minimum resolution required to provide a realistic value of the IBEAM current. The spatial resolution of the simulation grid in the diode region is $200 \mu \mathrm{m}$, which enables modeling of anode heating and bipolar flow. Plasma effects are not included and, instead, a simplified model of fluid particles is used to generate the falling diode impedance, if needed. In this model, massive electrons are injected from the anode surface after the pulse rise in order to attract a portion of the emitted ions. These electrons drift toward the cathode
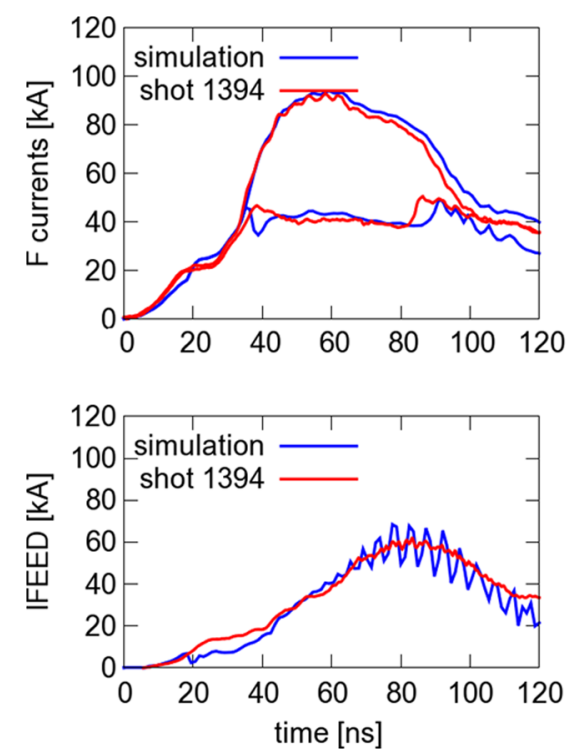

at $1 \mathrm{~cm} / \mu \mathrm{s}$ and, with accompanying ions, carry the anode potential into the AK gap, approximating an expanding anode plasma. After the initial "turn-on," the electron injection continues at the anode surface, increasing the plasma density. The appearance of the massive electrons at the anode interferes with the beam focus, however, so spot size and dose rate probes are not considered.

The rate of injection of the massive electrons is the main difference between the simulations. The only other difference is the voltage waveform injected at the boundary at $z=0$. The voltages used are plotted in Fig. 5 .

Explosive electron emission occurs in transmission lines when the local electric field stress exceeds the tolerance of the conductor $[14,15]$. The thresholds are a function of the material and its preparation. A typical values for accelerator cathodes range from 150 to $250 \mathrm{kV} / \mathrm{cm}$ [9]. The value may be determined by the departure of the transmission line current from their vacuum values, for example when $\mathrm{OF}$ exceeds IF. To achieve the best match the recent accelerator performance, the emission threshold in the MITL is $280 \mathrm{kV} / \mathrm{cm}$ over $0<z<115 \mathrm{~cm}$ and $100 \mathrm{kV} / \mathrm{cm}$ over $115<z<198.5 \mathrm{~cm}$. This reduction in threshold approximates the effect of pump-out ports near position $G$ (see Fig. 1) by allowing more electron emission where field enhancements occur. The field-shaper emission threshold is $950 \mathrm{kV} / \mathrm{cm}$, although for most simulations $550 \mathrm{kV} / \mathrm{cm}$ was sufficient to prevent emission. Electrons are emitted from the cathode needle above $100 \mathrm{kV} / \mathrm{cm}$, or $40 \mathrm{kV} / \mathrm{cm}$ where silver paint is applied.

\section{B. Shots at 3.5 MV}

One shot is modeled at the 3.5-MV endpoint voltage. This is shot 1394 which has an 8 -8 diode geometry. The simulated currents are compared to data in Fig. 6.
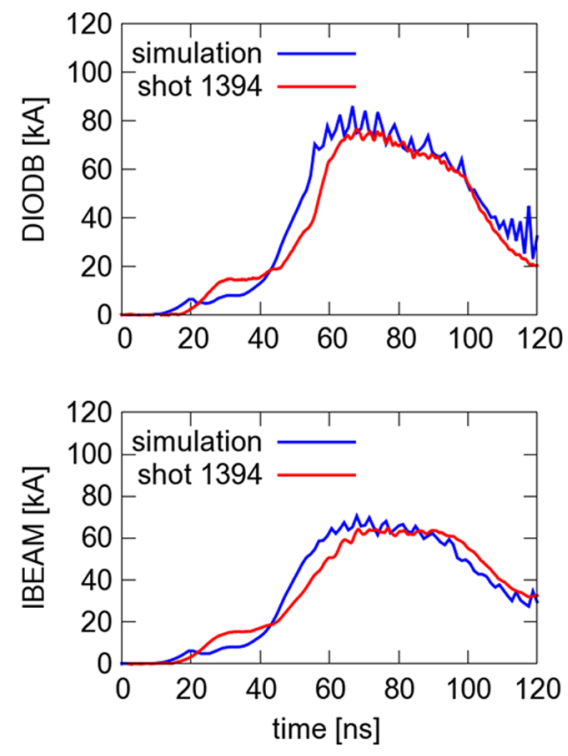

FIG. 6. Currents in the MITL, dustbin, and diode from a simulation of shot 1394 compared to data. The diode has a 8-mm diameter needle and a 8-mm AK gap. The diode impedance in the simulation is stable. 

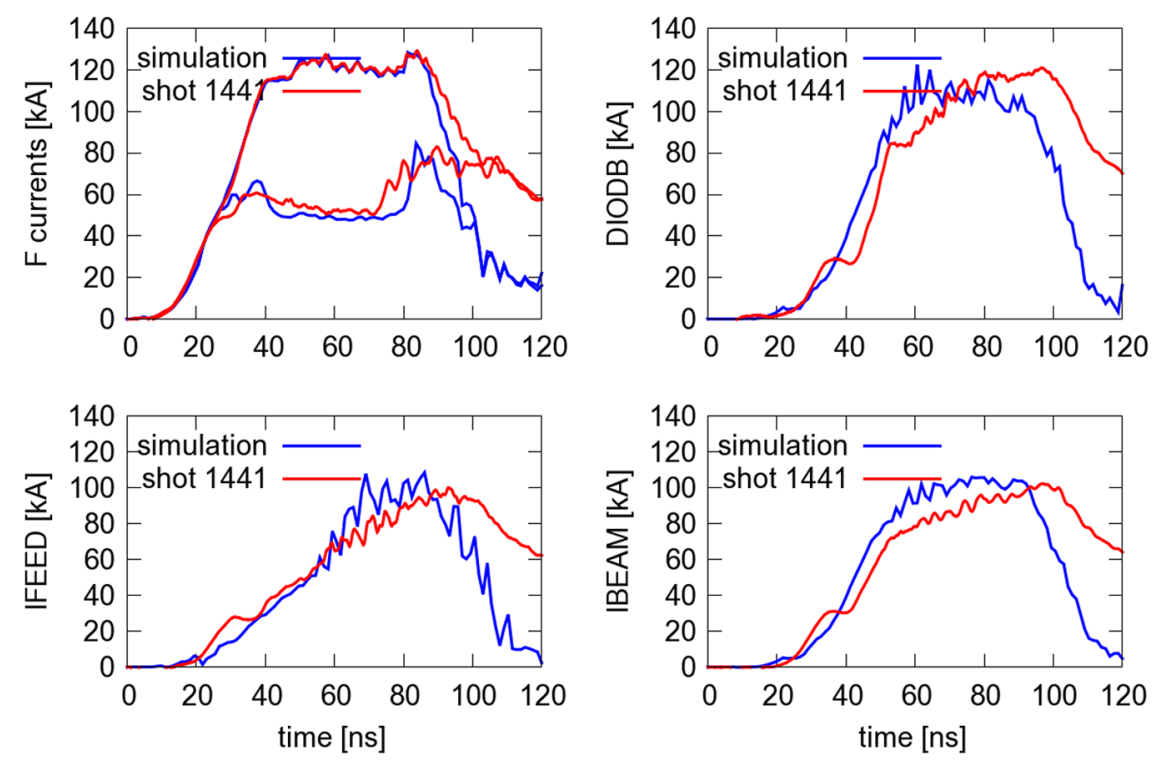

FIG. 7. Currents in the MITL, dustbin, and diode from a simulation of shot 1441 compared to data. The diode has a 7-mm diameter needle and a 7-mm AK gap.
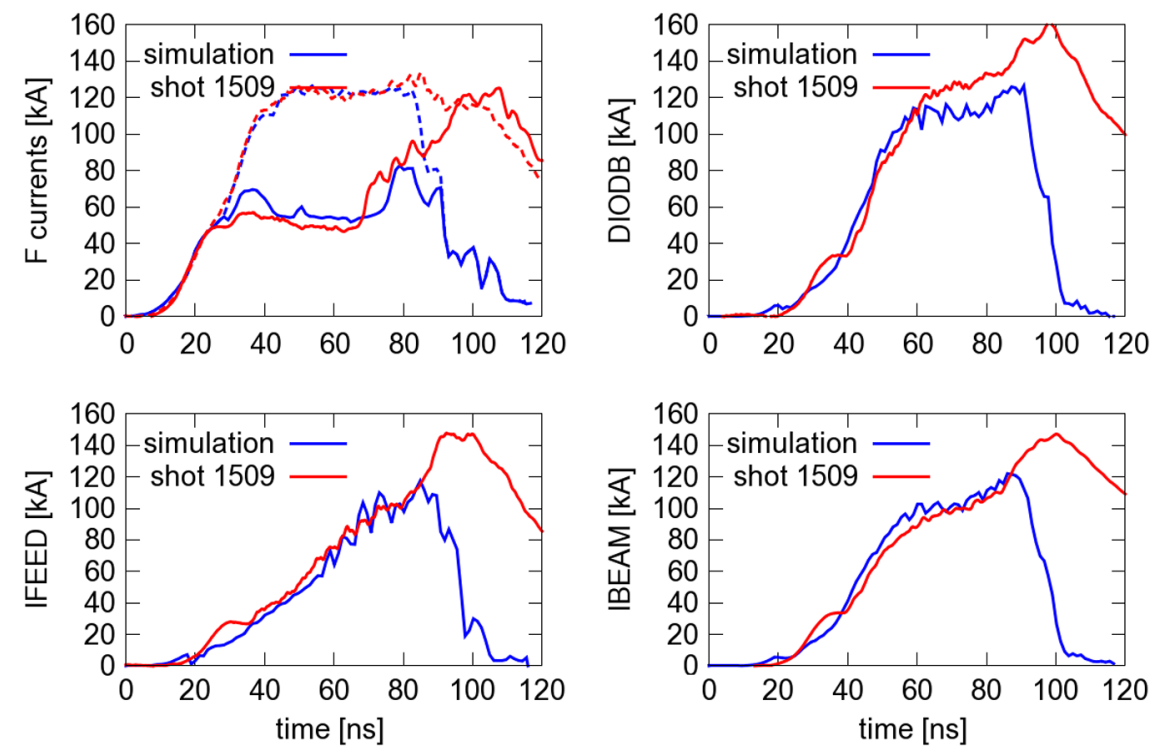

FIG. 8. Currents in the MITL, dustbin, and diode from a simulation of shot 1509 compared to data. The diode has a 7-mm diameter needle and a 7-mm AK gap. The falling diode impedance is modeled using massive fluid electrons at the anode which drift away from the surface and effectively reduce the AK gap.

All currents have smoothing applied, so no conclusion should be drawn regarding the magnitude of oscillations.

The retrapping speed on the field shaper is well matched, based on IFEED. Since DIODB equals IBEAM during the pulse rise, the sheath electrons are impacting the dustbin wall at larger radius. The rise in beam current is faster in simulation than data, and this causes the disagreement during the pulse rise for IBEAM and DIODB. The measured diode currents for all shots appear to turn-on rapidly in the first $10 \mathrm{~ns}$ and then relax to a more gradual rise. We do not attempt to model this effect.

\section{Shots at 4.5 MV}

Three shots are modeled for the 4.5-MV endpoint voltage (1441, 1509, 1519). Each uses the 7-7 geometry but all have different diode impedance lifetimes. The massivefluid-electron model is used in each simulation to achieve the falling diode impedance seen in the IBEAM current. 

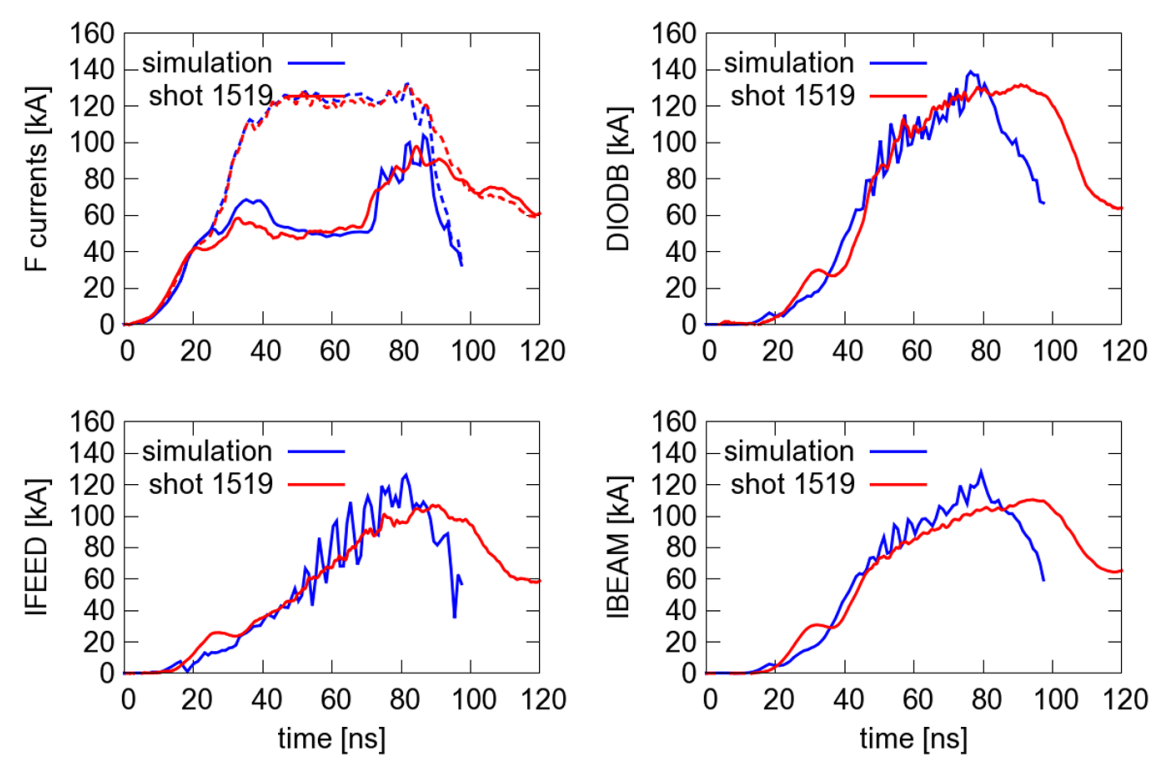

FIG. 9. Currents in the MITL, dustbin, and diode from a simulation of shot 1519 compared to data. The diode has a 7-mm diameter needle and a 7-mm AK gap. The falling diode impedance is modeled using massive fluid electrons at the anode which drift away from the surface and effectively reduce the AK gap.

The comparison to shot 1441 is shown in Fig. 7. A snapshot of the sheath electron density in the MITL for this simulation is shown in Fig. 4. The start time and rate of injection of massive electrons at the anode surface was adjusted to match the measured gap closure. The comparisons to shots 1509 and 1519 are shown in Figs. 8 and 9, respectively.

Comparing the IBEAM currents in Figs. 7, 8, and 9, we see a rapid decrease in diode impedance in shot 1509 . This shot begins identically to the others, but at approximately $30 \mathrm{~ns}$ into peak power, the diode current increases more rapidly. The reproducibility of the diode impedance profiles is examined with greater statistics in Sec. VI. The theory of the precollapse behavior is examined in Sec. VII.

\section{PULSED POWER CORRELATION TO POOR SHOTS}

Variations in injected power could manifest as differences in peak power or pulse rise, and these could impact sheath current uniformity or insulation. To determine if variations in peak power correlate with poor performing shots, we plot the peak anode current versus shot number for 99 shots in Fig. 10, noting which are good and poor. The peak anode current is calculated by taking the average value at $\mathrm{OF}$ during the first $10 \mathrm{~ns}$ of peak power. The average peak current is represented by a solid gray line and the standard deviation is indicated with dotted lines. We note that there is variation in the diode geometries in the shots in Fig. 10, but that we only attempt to identify a correlation with impedance fall and pulsed power.
The OF values of the good shots form a Gaussian distribution with mean $\bar{x}=121.8 \mathrm{kA}$ and standard deviation $\sigma=1.6 \mathrm{kA}$. The OF currents for poor shots, shown in red in Fig. 10, are well represented by this distribution, with the reduced $\chi^{2}=1 /(N-1) \times$ $\sum_{k}^{N}\left[\left(\bar{x}-x_{k}\right) / \sigma\right]^{2}=1.09$, until the retrapping wave arrives from downstream. There appears to be more of a correlation with shot number and peak current than with diode impedance.

The pulse rises for good shots are also normally distributed with $\bar{x}=24.6 \mathrm{~ns}$ and $\sigma=1.5 \mathrm{~ns}$. The pulse rises for poor shots, shown in red in Fig. 11, are again well represented by this distribution with reduced $\chi^{2}=1.33$.

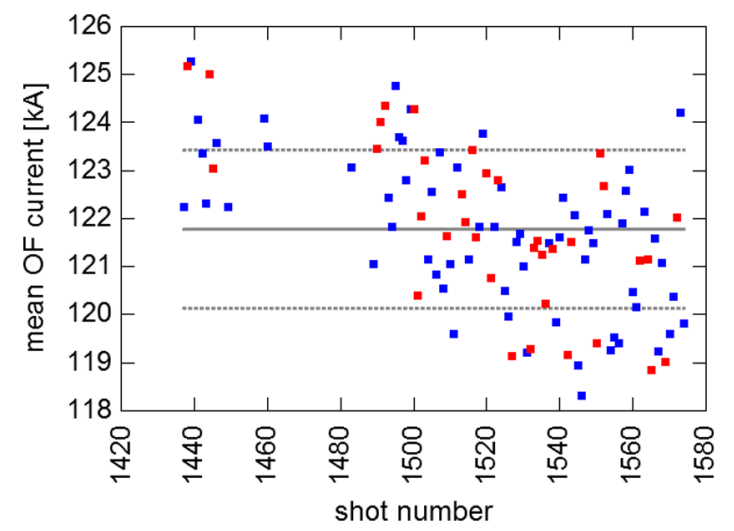

FIG. 10. The magnitude of the OF current at peak power versus shot number. Good shots are shown in blue and poor shots in red. The values shown are averaged over the first $10 \mathrm{~ns}$ at peak power. 


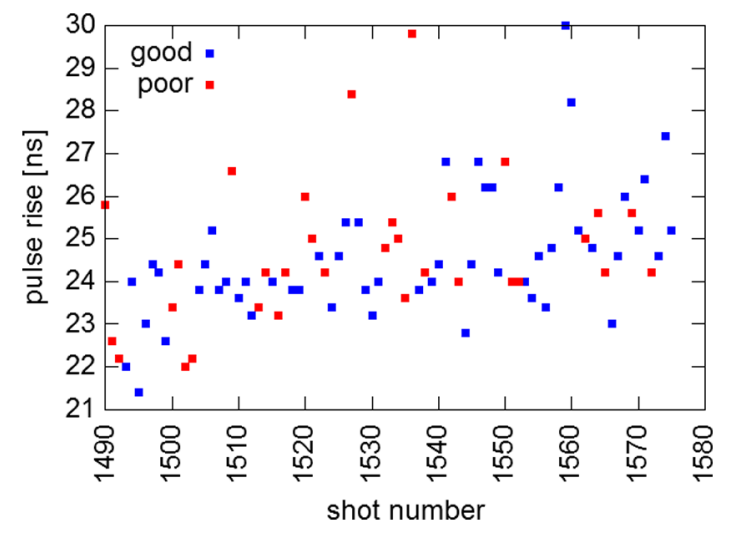

FIG. 11. The pulse rise from OF versus shot number. Good shots are shown in blue and poor shots in red. The rise is calculated as the time to rise from $10 \%$ to $90 \%$ of the mean OF current.

\section{DUSTBIN/MITL ALIGNMENT AND FIELD SHAPER CORRELATION TO POOR SHOTS}

If the cathode is misaligned to the anode in the transmission line or dustbin, the sheath current should become asymmetric around the line or field shaper. To determine if misalignment is correlated with poor shots, we calculate the asymmetries in the MITL and dustbin currents and
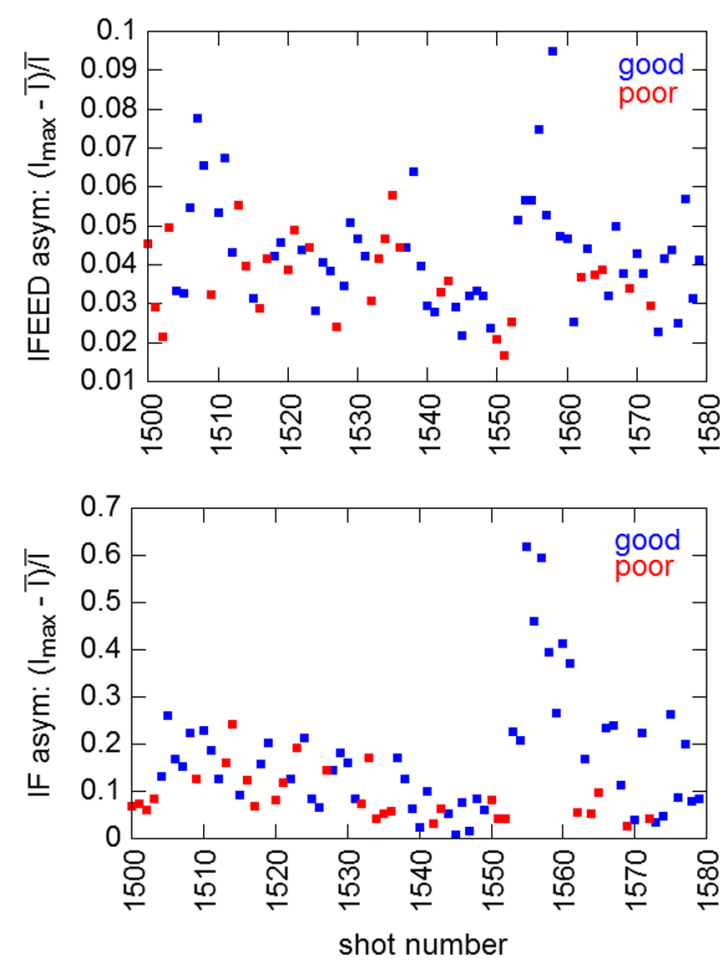

FIG. 12. The azimuthal asymmetry of the IFEED (top) and IF (bottom) currents versus shot number. The asymmetry is the difference between the highest probe value and the average, normalized by the average, $\left(I_{\max }-\bar{I}\right) / \bar{I}$. Good shots are shown in blue and poor shots in red. plot these values versus shot number, noting good and poor shots.

Each current presented in this report is the average of four B-dot probes arrayed azimuthally at a given axial position (listed in Sec. III). Azimuthal asymmetry is calculated as the difference between the highest probe value at a given position and the average, normalized by the average, $\left(I_{\max }-\bar{I}\right) / \bar{I}$. This value is calculated for the IFEED and IF probes from 80 shots and plotted versus shot number in Fig. 12. Good shots are shown in blue and poor shots in red. There is no discernible correlation between the level of asymmetry and shot performance. The IFEED and IF asymmetries for good shots are normally distributed with $\bar{x}=0.04$ and $\sigma=0.01$ for IFEED and $\bar{x}=0.18$ and $\sigma=0.14$ for IF. The values of the poor shots for IFEED and IF asymmetry are well represented by these distributions (reduced $\chi^{2}=0.69$ and 0.60 , respectively.)

There are six field shapers used with the small dustbin which have very modest differences in curvature shape and surface damage. Although one field shaper has a higher fraction of poor shots than the others, there are poor shots are recorded for each at least $46 \%$ of the time. Therefore, we do not conclude that the shots fail exclusively as a result of a subset of field shaper hardware.

\section{DIODE IMPEDANCE COLLAPSE CHARACTERISTICS}

In the compilation of cathode and anode currents (IF and OF) in Sec. II, poor shots are identified by a larger-thanaverage rise in IF after the retrapping wave has passed. This is a result of a larger-than-average drop in the load impedance. Since the abnormal current increase is first recorded in the IBEAM probes, the impedance change must be occurring in the diode, and not elsewhere in the dustbin.

In normal operation, the SMP diode has a slowly decreasing impedance $(<0.5 \Omega / \mathrm{ns})$ related to anode plasma expansion. This results in a steady rise in the IBEAM currents, after the pulse rise, as shown in Figs. 13 and 14. Initially, the rate of increase is the same for all shots and is related to $r_{c} / g$, as discussed in Sec. VII. For poor shots, IBEAM increases sharply at some time during peak power. The time to impedance breakdown is different for the shots in this series, but breakdown is never gradual. The drop in impedance is catastrophic, causing the diode to draw the entire line current.

In average operation, the DIODB current is higher than IBEAM after pulse rise. This indicates that some sheath current is wrapping around the field shaper and connecting to the anode within an 11-cm radius. (This is the electron density seen on the anode holder in Fig. 4.) In this shot series, when the diode impedance drops abnormally low, IBEAM and DIODB rise to nearly the same value and IF becomes almost equal to $\mathrm{OF}$, indicating that the power flow is recaptured in the cathode and the diode current is almost 

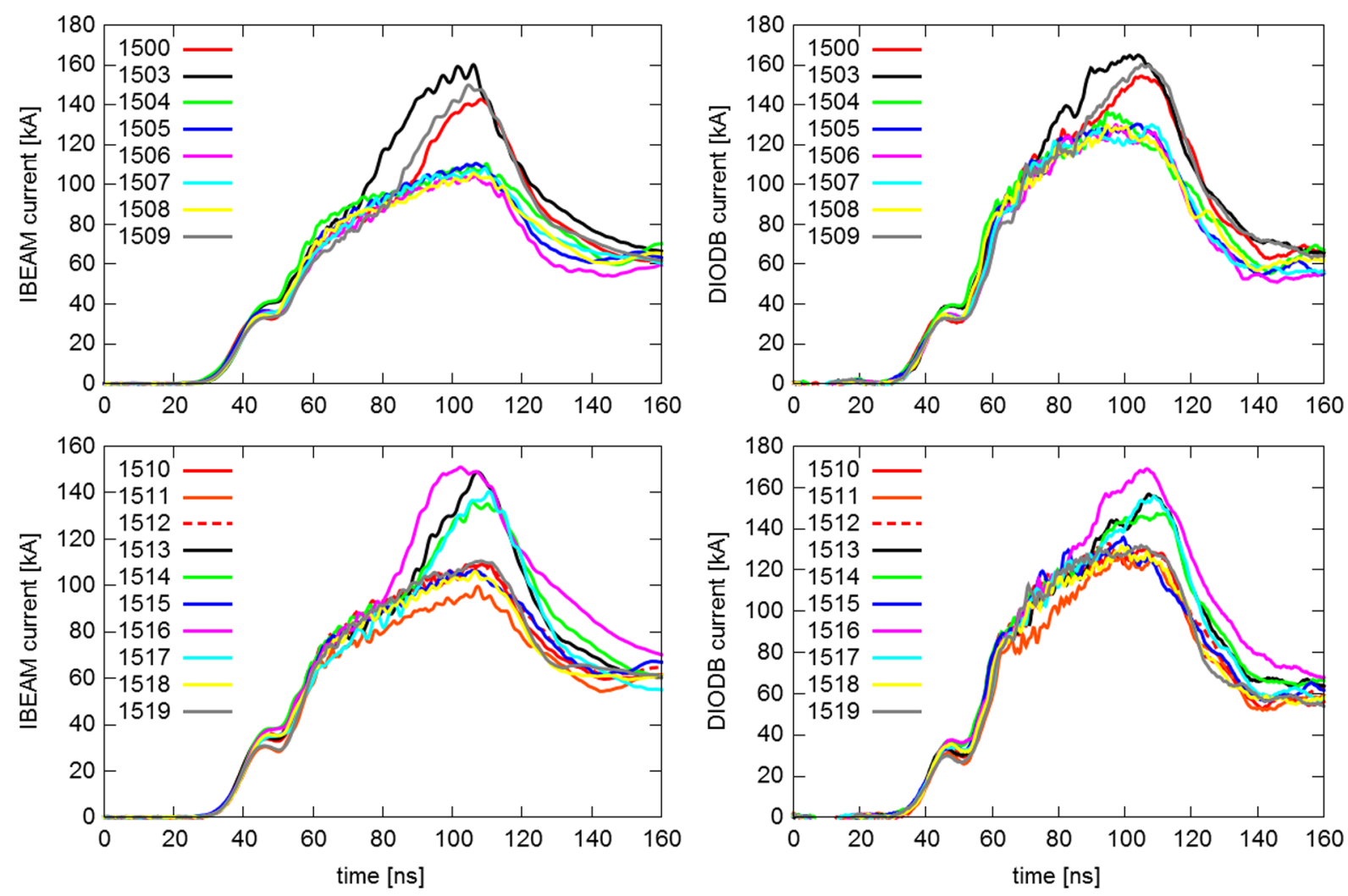

FIG. 13. The IBEAM and DIODB currents from shots 1500 through 1519. All shots are 7-7.
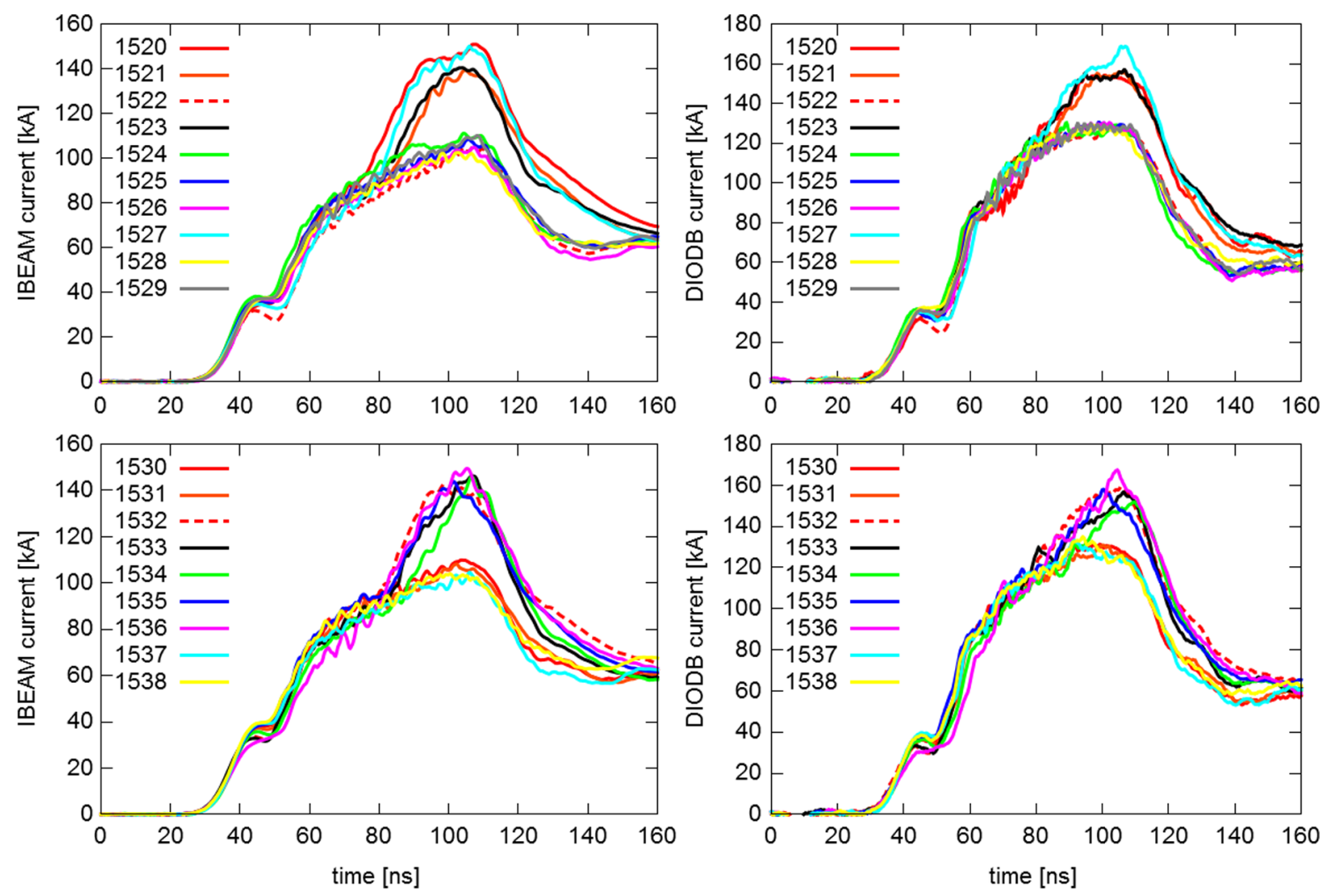

FIG. 14. The IBEAM and DIODB currents from shots 1520 through 1539. All shots are 7-7. 


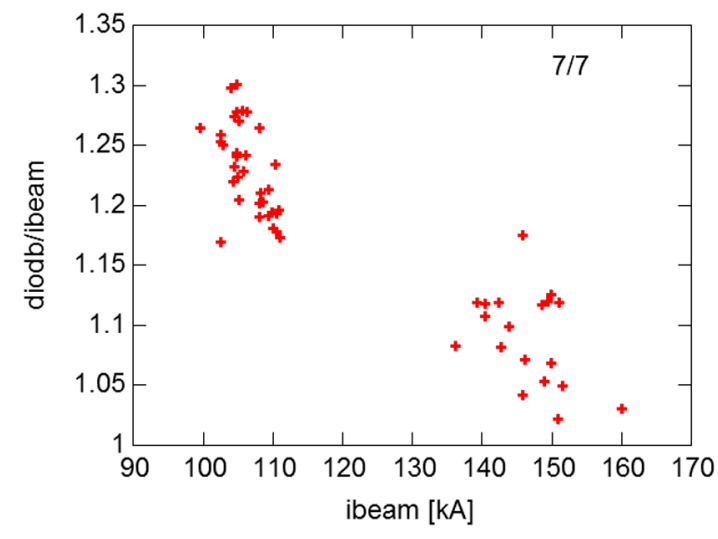

FIG. 15. IBEAM versus the ratio DIODB/IBEAM from 53 7-7 shots. The peak currents are used.

the total current in the system. This is seen in the IBEAM and DIODB currents for the 7-7 shots in Figs. 13 and 14, and in the IF/OF currents in Fig. 3.

To quantify how much the sheath current near the load is retrapped for poor shots, the ratio of DIODB to IBEAM is plotted versus IBEAM in Fig. 15. We see that there are two distinct distributions. For good shots, IBEAM ranges around 100 to $110 \mathrm{kA}$ and DIODB is $16 \%$ to $30 \%$ larger. For failed shots IBEAM is near 140 to $150 \mathrm{kA}$ and DIODB is usually less than $10 \%$ larger. That the distribution is bimodal instead of continuous and involves a significant reduction in sheath current strongly suggests that sheath electrons are not contributing to this catastrophic impedance collapse, but rather this phenomenon is a result of physics in the diode region.

\section{DIODE CURRENT COMPARISON TO THEORY}

The SMP diode operates as a current-limited device, in which the critical current is given by $[3,16]$

$$
I_{\text {crit }}=8.5 \alpha \frac{r_{C}}{g}\left(\gamma^{2}-1\right)^{1 / 2}[\mathrm{kA}]
$$

where $\gamma=1+e V / m_{e} c^{2}, r_{C}$ is the cathode radius, $g$ is the AK gap width, and $\alpha$ is a scale factor used to account for the current increase due to ion space-charge. Estimates of $\alpha$ range from 1.6 to 2.8 , depending on the diode geometry and the beam and ion space-charge profiles. Therefore, Eq. (1) includes bipolar flow, but does not include the effects of evolving plasmas as described in Ref. [10]. Two plasmarelated effects were identified which decrease the diode impedance during operation. The first is an expanding anode plasma, which continually reduces the effective $g$ and occurs in normal diode operation. The second is an increased ion space-charge around the cathode needle, which is represented as an increase in $\alpha$.

Equation (1) indicates that diodes with the same $r_{C} / g$ will have the same impedance. This should be seen in comparisons of IBEAM $\left(I_{\text {crit }}\right)$ early in the pulse for shots at the same voltage $(\gamma)$. However, since the plasma expansion rate is a function of the diode voltage, the rate of gap closure, $g(t)$, will be the same for all shots. Therefore, the ratio $r_{C} / g(t)$ varies differently as $g(0)$ changes, even as $r_{C} / g(0)$ and voltage are not varied. If we denote the plasma expansion velocity as $v_{p}$, the gap closure rate can be modeled simply as $g(t)=g_{0}-v_{p} t$. Then

$$
I_{\text {crit }}(t)=I_{\text {crit }}(0) \frac{g_{0}}{g_{0}-v_{p} t},
$$

independent of $r_{C}$, for constant $\gamma$.

To recover the equivalence of shots with identical $r_{C} / g$, but different $g$, the diode impedance can be plotted on a scaled time axis using

$$
I_{\text {crit }}\left(t^{\prime}\right)=I_{0}\left(\frac{1}{1-v_{p} t^{\prime}}\right)
$$

where $t / g_{0} \rightarrow t^{\prime}$, assuming $\gamma$ is constant, or

$$
Z_{\text {diode }}\left(t^{\prime}\right)=Z_{0}\left(1-v_{p} t^{\prime}\right)
$$

if it is not.

Simulation results for $Z_{\text {diode }}\left(t^{\prime}\right)$ are plotted in Fig. 16, which compares an 8.5-mm-diameter cathode to a $12.5-\mathrm{mm}$ cathode, both with $r_{C} / g_{0}=2$. Expanding anode plasmas are included in the model.

Measurement results for $I_{\text {crit }}\left(t^{\prime}\right)$ are shown in Fig. 17. The diode currents from three random shots, a 6-6, 7-7, and $8-8$, are compared. $\gamma(t)$ is assumed to be a similar function for each shot. Figure 17(a) shows IBEAM currents on an unscaled time axis, while Fig. 17(b) shows the same data with $t^{\prime}=t / g_{0}$.

Since the scaling of Eq. (3) holds for simulation and data, we can determine the value of $v_{p}$. In Fig. 18, Eq. (1) is plotted over IBEAM from shot 1441, using $v_{p}=1 \mathrm{~cm} / \mu \mathrm{s}$,

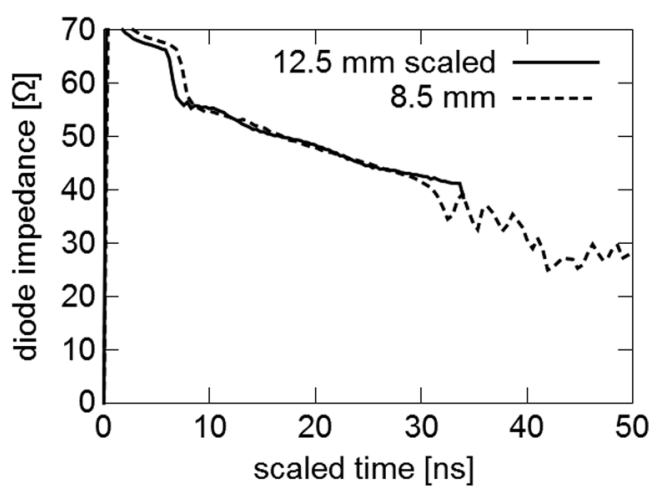

FIG. 16. The diode impedance from simulations of 8.5 and 12.5-mm diameter cathodes on a scaled time axis. (For the 12.5-mm cathode, $t \rightarrow 8.5 / 12.5 t$.) The simulations include effects of expanding anode plasmas. 


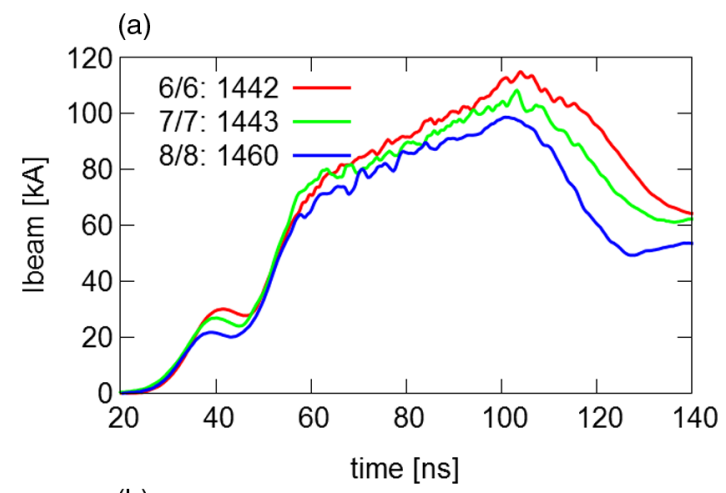

(b)

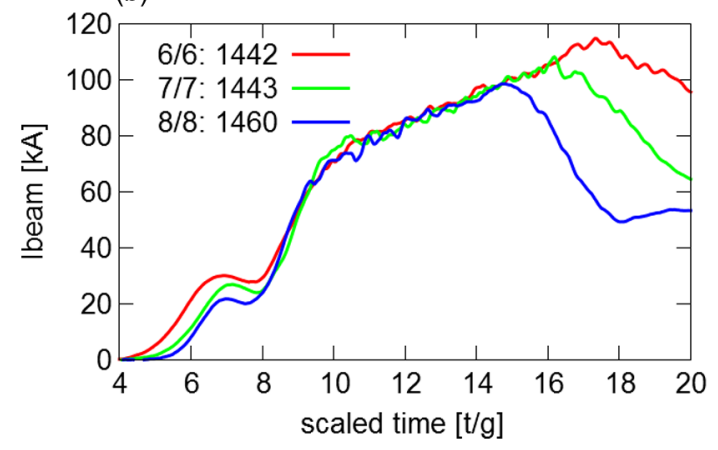

FIG. 17. (a) The IBEAM diode currents from shots 1442, 1443, and 1460, which are 6-6, 7-7, and 8-8 configurations, respectively. (b) The same IBEAM currents with the time axes scaled by $t^{\prime}=t / g_{0}$.

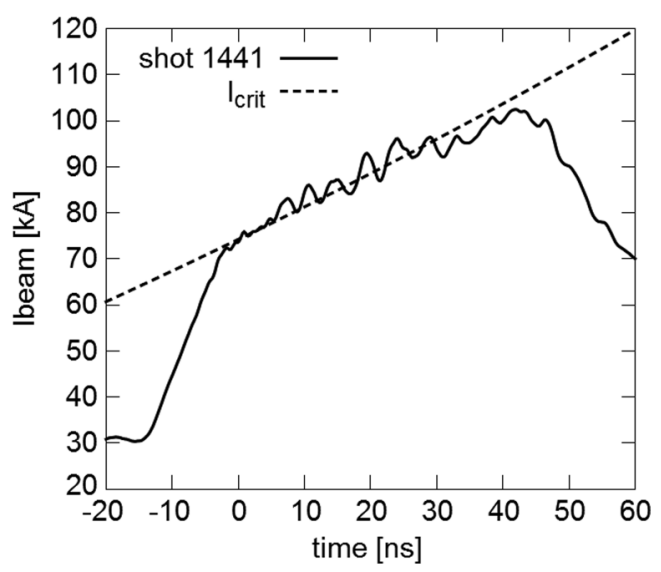

FIG. 18. Theoretical critical current and IBEAM for a timedependent AK gap. Equation (1) using $v=1 \mathrm{~cm} / \mu \mathrm{s}, \alpha=1.1$, and $\gamma(t)=8+0.0625 t$ where $t$ is in ns. The time axis has been shifted.

$\alpha=1.1$, and $\gamma(t)=8+0.0625 t$, where $t$ is in ns. The form of $\gamma(t)$ is estimated from the simulation in Sec. III C. We see that the $v_{p}$ used in the massive-electron-model and Eq. (1) are the same.

\section{CONCLUSION}

The recent experimental campaign conducted on Sandia's RITS-6 accelerator was used to improve understanding of the shot-to-shot variation of the SMP diode. The accelerator was operated at 4.5 MV which simplified the analysis by removing the variability introduced by field shaper emission.

In this paper, we examined sources of variability which occur away from the diode, specifically (i) sheath electron emission and trajectory changes, (ii) variations in pulsed power amplitude, (iii) variations in pulse rise, (iv) dustbin and transmission line misalignment, and (v) different field shapers. Simulations of the transmission line, dustbin, and diode showed consistent sheath electron emission and trajectories. Statistical analyses of peak power, pulse rise, asymmetries in IFEED, and asymmetries in IF show no correlation with poor radiographic shots. Lastly, one of the six field shapers used has a higher occurrence of shot failure, however failures occur for each. We find no sources of accelerator variation which are associated with poor radiographic performance.

Shots which perform well radiographically are well described by the theoretical critical current equation in which we use a time-dependent AK gap width. The estimated gap closure velocity is approximately $v_{p}=1 \mathrm{~cm} / \mu \mathrm{s}$.

In classifying good versus poor shots, we find that there is not a continuous spectrum of diode impedance behavior but that the good and poor shots can be grouped into two distinct impedance profiles. This result will form the basis of a follow-up study focusing on the variability resulting from diode parameters and beam and plasma physics.

\section{ACKNOWLEDGMENTS}

This work was conducted by National Security Technologies, LLC, under Contract No. DE-AC5206NA25946 with the U.S. Department of Energy. This work was also conducted by Sandia National Laboratories for the U.S. Department of Energy's National Nuclear Security Administration under Contract No. DE-AC0494AL85000.

[1] J. Threadgold, I. Crotch, D. Rose, and J. Maenchen, in Proceedings of the 14th IEEE International Pulsed Power Conference (IEEE, Piscataway, NJ, 2003), p. 995.

[2] K. D. Hahn, N. Bruner, S. Cordova, R. Gignac, M. D. Johnston, J. Lechbee, I. Molina, B. V. Oliver, S. Portillo, J. Threadgold, T. J. Webb, D. R. Welch, and D. Ziska, IEEE Trans. Plasma Sci. 38, 2652 (2010).

[3] S. Swanekamp, G. Cooperstein, J. Schumer, D. Mosher, F. Young, P. F. Ottinger, and R. Commisso, IEEE Trans. Plasma Sci. 32, 2004 (2004).

[4] D. Hinshelwood, G. Cooperstein, D. Mosher, D. Ponce, S. Strasburg, S. Swanekamp, S. Stephanakis, B. Weber, F. Young, A. Critchley, I. Crotch, and J. Threadgold, IEEE Trans. Plasma Sci. 33, 696 (2005).

[5] M. D. Johnston, B. V. Oliver, S. Portillo, J. E. Maenchen, T. A. Mehlhorn, D. R. Welch, D. V. Rose, N. Bruner, 
D. Droemer, Y. Maron, and A. D. Heathcote, in Proceedings of the 34th IEEE International Conference on Plasma Science (IEEE, Piscataway, NJ, 2007), p. 445.

[6] P. Martin, J. Threadgold, and S. Vickers, IEEE Trans. Plasma Sci. 41, 2510 (2013).

[7] I. Smith, V. L. Bailey, J. Fockler, J. S. Gustwiller, D. L. Johnson, J. E. Maenchen, and D. W. Droemer, IEEE Trans. Plasma Sci. 28, 1653 (2000).

[8] D. Johnson, V. Bailey, R. Altes, P. Corcoran, I. Smith, S. Cordova, K. Hahn, J. Maenchen, I. Molina, S. Portillo, E. Puetz, M. Sceiford, D. V. de Valde, D. Rose, B. Oliver, D. Welch, and D. Droemer, in Proceedings of the 15th IEEE International Pulsed Power Conference (IEEE, Piscataway, NJ, 2005), p. 314.

[9] N. Bruner, T. Genoni, E. Madrid, D. Rose, D. Welch, K. Hahn, J. Leckbee, S. Portillo, B. Oliver, V. Bailey, and
D. Johnson, Phys. Rev. ST Accel. Beams 11, 040401 (2008).

[10] N. Bruner, D. R. Welch, K. D. Hahn, and B. V. Oliver, Phys. Rev. ST Accel. Beams 14, 024401 (2011).

[11] J. M. Creedon, J. Appl. Phys. 46, 2946 (1975).

[12] C. W. Mendel, J. Appl. Phys. 50, 3830 (1979).

[13] D. Rose, D. R. Welch, B. V. Oliver, R. E. Clark, D. L. Johnson, J. E. Maenchen, P. R. Menge, C. L. Olson, and D. C. Rovang, J. Appl. Phys. 91, 3328 (2002).

[14] I. Langmuir and K. Blodgett, Phys. Rev. 22, 347 (1923).

[15] R. Fowler and L. Nordheim, Proc. R. Soc. A 119, 173 (1928).

[16] B. V. Oliver, K. D. Hahn, M. D. Johnston, and S. Portillo, Acta Phys. Pol. A 115, 1044 (2009). 